\title{
The anti-fibrotic effect of human fetal skin- derived stem cell secretome on the liver fibrosis
}

\author{
Xia Yao', Jing Wang ${ }^{2}$, Jiajing Zhu ${ }^{3}$ and Xiaoli Rong ${ }^{4,5^{*}}$
}

\begin{abstract}
Background: Liver fibrosis resulting from chronic liver injury is one of the major causes of mortality worldwide. Stem cell-secreted secretome has been evaluated for overcoming the limitations of cell-based therapy in hepatic disease, while maintaining its advantages.

Methods: In this study, we investigated the effect of human fetal skin-derived stem cell (hFSSC) secretome in the treatment of liver fibrosis. To determine the therapeutic potential of the hFSSC secretome in liver fibrosis, we established the $\mathrm{CCl}_{4}$-induced rat liver fibrosis model and administered hFSSC secretome in vivo. Moreover, we investigated the anti-fibrotic mechanism of hFSSC secretome in hepatic stellate cells (HSCs).

Results: Our results showed that hFSSC secretome effectively reduced collagen content in liver, improved the liver function and promoted liver regeneration. Interestingly, we also found that hFSSC secretome reduced liver fibrosis through suppressing the epithelial-mesenchymal transition (EMT) process. In addition, we found that hFSSC secretome inhibited the TGF- $\beta 1$, Smad2, Smad3, and Collagen I expression, however, increased the Smad7 expression.
\end{abstract}

Conclusions: In conclusions, our results suggest that hFSSC secretome treatment could reduce $\mathrm{CCl}_{4}$-induced liver fibrosis via regulating the TGF- $\beta / S$ mad signal pathway.

Keywords: hFSSC, Secretome, Liver fibrosis, TGF- $\beta /$ Smad

\section{Introduction}

Liver fibrosis is a wound healing response generated against chronic or iterative liver injury [1]. Recent evidence suggests that stem cell-based liver fibrosis treatment can be mediated through paracrine effects $[2,3]$. The exclusive use of stem cell-secreted secretome has been evaluated for overcoming the limitations of cell-based therapy, while maintaining its advantages to their parent cells [4].

\footnotetext{
*Correspondence: rongx116@mails.ju.edu.cn; xrong5@jhmi.edu

${ }^{4}$ Department of Clinical Laboratory, The Affiliated Hospital of Changchun University of Chinese Medicine, 1478 Gongnong Road, Changchun 130021, Jilin, China

${ }^{5}$ Neuroregeneration and Stem Cell Programs, Institute for Cell Engineering, The Johns Hopkins University School of Medicine, Baltimore, MD 21205, USA Full list of author information is available at the end of the article
}

It included extracellular vesicles and other soluble proteins or biologically active molecules. In addition, previous studies have indicated that human bone marrow mesenchymal stem cell-derived exosomes and other stem cellderived secretome can reduce liver fibrosis $[5,6]$.

Previous studies have indicated that the features of fetal tissue cells facilitate engraftment in vivo and may provide preferred effects against diseases difficult to treat [7]. Since 1928, hundreds of clinical trials using various types of fetal transplants have been performed worldwide [8-10]. Moreover, recent studies have demonstrated that human fetal stem cells (FSCs) have a great growth promoting potential, which benefited to the tissue regeneration and cell therapy $[11,12]$. In 
comparison to other mesenchymal stem cell (MSCs), FSCs are easier to culture and more readily proliferate and less likely to be rejected by transplant recipients, as these cells are less antigenic [8]. Although there remain still ethical and social issues with respect to the clinical use of fetal tissue, fetal stem cell secretome transplantation may overcome these problems and have more perspectives on hepatic disease treatment.

In our previous study, we have successfully isolated and identified hFSSCs [13]. Interestingly, we found hFSSC secretome has great ability to control and balance the collagen formation in skin tissue [13]. Therefore, we hypothesized that hFSSCs have a potential to reduce the collagen formation in liver fibrosis, whereas liver fibrosis is caused by over-abundance of collagen. We further analyzed the effects of hFSSC secretome on live fibrosis in vitro and investigated the mechanism of hFSSC secretome and TGF- $\beta /$ Smad pathway involvement on anti-fibrosis. Our preliminary results provide the first evidence that hFSSC secretome effectively reduce liver fibrosis through the TGF- $\beta /$ Smad pathway. We believe that the hFSSC secretome as an acellular regenerative therapy and approaches can provide great potential for the treatment of liver fibrosis.

\section{Materials and methods \\ Cell culture}

hFSSCs and human umbilical cord mesenchymal stem cell (hUCMSCs) were provided and extracted by our previous study [13]. HSCs were purchased from the Chinese Academy of Medical Sciences, China. In brief, hFSSCs, hUCMSCs, and HSCs were cultured in high glucose DMEM (Gibco, Grand island, USA) supplemented with $500 \mathrm{U} / \mathrm{ml}$ penicillin and $500 \mu \mathrm{g} / \mathrm{ml}$ streptomycin (Invitrogen, Shanghai, China), and 10\% FBS (Gibco, Grand island, USA) at $37^{\circ} \mathrm{C}$, with saturated humidity and $5 \% \mathrm{CO}_{2}$. hFSSCs and hUCMSCs at the P5 were used for this study. hFSSC and hUCMSC secretome was collected as reported in our previous study [13]. Briefly, cells were cultured and reached 70 80\% confluence, then placed in serum-free medium (SFM; Invitrogen, Shanghai, China), added with $5 \mathrm{ml}$ of 200 mM L-glutamine solution (Invitrogen, Shanghai, China) in $500 \mathrm{ml} \mathrm{SFM}$ prior to use, and incubated in $5 \% \mathrm{CO}_{2}$ in a humidified condition. After being cultured $24 \mathrm{~h}$, the conditioned medium (CM) was collected and centrifuged to purify for $10 \mathrm{~min}$ at $4{ }^{\circ} \mathrm{C}, 4000 \mathrm{~g}$. Next, 10-ml conditioned medium was re-centrifuged with Amicon Ultra Centrifugal Filters (Millipore Corp, Billerica, MA, USA) at $4{ }^{\circ} \mathrm{C}, 4000 \mathrm{~g}, 2 \mathrm{~h}$. At last, 300 500 $\mu$ l supernatant solution was collected as cell-free secretome each time. The protein content was measured using the BCSA kit
(Thermo Scientific, Rockford, IL, USA) according to the manufacturer's instruction.

\section{$\mathrm{CCl}_{4}$-induced liver fibrosis in rats}

Liver fibrosis was induced in Sprague Dawley (SD) rats (8-week-old, female, $200 \mathrm{~g}$ ). All protocols and procedures were approved by the Animal Experiment Ethic Committee of Changchun University of Traditional Chinese Medicine (Approval NO. XW201903167). All experimental procedures were in accordance with the Chinese legislation regarding experimental animals. Detailed procedures for $\mathrm{CCl}_{4}$-induced have been described in our published studies [6]. Briefly, rats were administered with an intraperitoneal injection of $30 \% \mathrm{CCl}_{4}, 3$ $\mathrm{ml} / \mathrm{kg}$ body weight twice weekly in olive oil. After 8 weeks, $\mathrm{CCl}_{4}$-treated rats were randomly assigned into three groups ( $n=10$ rats, tail vein injection/weekly): PBS group $(1 \mathrm{ml})$, hUCMSC secretome group $(250 \mu \mathrm{g}, 1 \mathrm{ml})$, and hFSSC secretome group $(250 \mu \mathrm{g}, 1 \mathrm{ml})$. After 4 weeks, liver tissue and serum were collected. Livers were divided into two parts of preservation in $10 \%$ formalin and freezing at $-80^{\circ} \mathrm{C}$.

\section{Histopathological analysis}

Liver tissues were processed for paraffin embedding by slicing into $4-\mu \mathrm{m}$ sections. Liver sections were stained with hematoxylin and eosin (H\&E), Masson, and Sirius red according to standard protocols. We selected the liver section fields randomly to analyze the liver fibrosis. The percentage of collagen-stained area was calculated via Image-Pro Plus. Immunohistochemistry (IHC) was measured with the Kit (Maixin KIT-9710, Fuzhou, China) in accordance with the manufacturer's instructions. In brief, the liver sections were deparaffinized, rehydrated, and incubated in a $99^{\circ} \mathrm{C}$ water bath for $15 \mathrm{~min}$. Then, the sections were incubated with $3 \% \mathrm{H}_{2} \mathrm{O}_{2}$ for $15 \mathrm{~min}$ and blocked with $10 \%$ normal goat serum (Sigma, USA) for $1 \mathrm{~h}$ at $37^{\circ} \mathrm{C}$, following with the incubation of primary antibody against PCNA (ab15497, 1:500 dilution, Abcam, Cambridge, UK), $\alpha$-SMA (ab5694, 1:500 dilution, Abcam, Cambridge, UK), and HNF-4 $\alpha$ (ab219610, 1:500 dilution, Abcam, Cambridge, UK) overnight at $4{ }^{\circ} \mathrm{C}$. Next, sections were incubated with biotinylated goat-anti-rabbit IgG antibody, added with diaminobenzidine solution for $15 \mathrm{~min}$ at $37^{\circ} \mathrm{C}$, then incubated with avidin peroxidase reagent, and hematoxylin for counterstaining. Lastly, slides were photographed using an optical microscope (Olympus, Tokyo Metropolitan, Japan). We used 10 random fields per section and 10 sections in total ( $n=10$ rats) for quantification of IHC results. The IHC results were calculated via Image-Pro Plus.

\section{Biochemical analysis}

The serum levels of alanine aminotransferase (ALT), aspartate aminotransferase (AST), total protein (TP), total 
bilirubin (TBIL), alkaline phosphatase (ALP), and gamma glutamyl transpeptidase $(\gamma-G T)$ were assessed using the Automated Biochemical Analyzer (AU-680, Beckman, California, USA) according to the procedure. Liver homogenate $(10 \%, \mathrm{w} / \mathrm{v})$ was prepared by homogenizing the right lobe of liver on ice in $150 \mathrm{mM}$ Tris- $\mathrm{HCl}$ buffered saline ( $\mathrm{pH}$ 7.2) using a polytron homogenizer (PT3100D; Kinematical, Lucerne, Switzerland). The levels of malondialdehyde (MDA) and hydroxyproline (Hyp) in liver tissue were measured using kits (Nanjing JianCheng Bio., Nanjing, China) according to the manufacturer's instructions.

\section{Quantitative real-time PCR (RT-qPCR)}

HSCs were co-cultured with either PBS, hUCMSC secretome, or hFSSC secretome $(5 \mathrm{ng} / \mathrm{ml})$ for $48 \mathrm{~h}$ before samples were collected for mRNA extraction. Total RNA was isolated from HSCs using Trizol reagent (Invitrogen, Shanghai, China) according to the manufacturer's protocol. Then, $1 \mu \mathrm{g}$ total RNA was reverse-transcribed to give cDNA, which was used as the template, and combined with standard SYBR premix Ex Taq (Invitrogen, Shanghai, China) on the qPCR Detection System (Roche, Basel, Switzerland), and experiments were conducted in triplicate. The levels of EMT-related genes (E-cadherin, Snail1, Vimentin, FSP1, and $\alpha$-SMA), TGF- $\beta /$ Smad signaling pathway-related genes (TGF- $\beta 1$, Smad2, Smad3, Smad7 and Collagen I), and the internal standard GAPDH mRNA were measured by RT-qPCR. The primers are listed in Table S1, and GAPDH served as the internal control. All reactions were performed in triplicate, and the data were analyzed using the $2^{-\Delta \Delta \mathrm{Ct}}$ method.

\section{Immunofluorescence (IF) staining}

When HSCs reached $60 \sim 70 \%$ confluence on 24-well plates, they were cultured with either PBS, hUCMSC secretome, or hFSSC secretome $(5 \mathrm{ng} / \mathrm{ml})$ for $48 \mathrm{~h}$. Next, HSCs were incubated with $4 \%$ paraformaldehyde at room temperature for $10 \mathrm{~min}$ and then incubated with $1 \%$ bovine serum albumin (BSA, Biosharp, Wuhan, China) for $30 \mathrm{~min}$. Cells were incubated with a primary antibody against $\alpha$-SMA (ab5694, 1:100 dilution, Abcam, Cambridge, UK) for $1 \mathrm{~h}$, followed by incubation with a secondary antibody (goat anti-rabbit IgG, ab15007, 1:500 dilution, Abcam, Cambridge, UK) for $30 \mathrm{~min}$ at room temperature. Rhodamine phalloidin (Thermal Scientific, Waltham, USA) was stained for cytoskeleton. The nuclei were labeled with DAPI (Thermal Scientific, Waltham, USA). Fluorescent images were captured using an EVOS Cell Imaging System (Thermo Scientific, Waltham, USA).

\section{Western blotting}

HSCs were co-cultured with either PBS, hUCMSC secretome, or hFSSC secretome $(5 \mathrm{ng} / \mathrm{ml})$ for $48 \mathrm{~h}$ before samples were collected for protein extraction. Protein samples were mixed with SDS sample buffer and heated to $95^{\circ} \mathrm{C}$ for $10 \mathrm{~min}$, followed by separation on SDSpolyacrylamide gels. Resolved proteins were electroblotted onto nitrocellulose membrane and probed with antibodies against TGF- $\beta 1$ (ab92486), Smad2 (ab40855), Smad3 (ab40854), Smad7 (ab216428), Collagen I (ab90395), and $\beta$-actin (ab5694), (1:1000 dilution, Abcam, Cambridge, UK) overnight at $4{ }^{\circ} \mathrm{C}$ (1:1000 dilution, Abcam, Cambridge, UK). Nitrocellulose membranes were then incubated with a secondary antibody, HRP-conjugated goat anti-rabbit IgG (ab15007), at room temperature for $2 \mathrm{~h}$, and visualized by chemiluminescent detection according to the manufacturer's instructions (Immobilon western chemiluminescent HRP substrate, Millipore, Massachusetts, USA).

\section{Statistical analysis}

Statistical analysis was performed using GraphPad Prism Version 6. One-way ANOVA with Dunnett's multiple comparisons test was used to test for statistically significant differences. All quantitative data are expressed as mean \pm SD for at least three independent experiments, and $p<0.05$ was considered to be statistically significant.

\section{Results}

\section{hFSSC secretome reduced $\mathrm{CCl}_{4}$-induced liver fibrosis in} rats

To explore the effect of hFSSC secretome on liver fibrosis, we used $\mathrm{CCl}_{4}$-induced liver fibrosis model in rats (Fig. 1a). Compared to the PBS group, gross morphology changes obviously in the hFSSC secretome group, including less fibrous nodular and more ruddy on the surface, more uniform surface, and soft texture (Fig. 1b). After 4 weeks' treatment, histopathological analysis using Masson and Sirius red staining indicated that the collagen area percentage in the hFSSC group (9.2\%) was significantly reduced, compared to the other two control groups $(24.3 \%$ in the PBS group and $14.9 \%$ in the hUCMSC secretome group, Fig. $1 \mathrm{~b}$ and $\mathrm{c}, p<0.05)$. Furthermore, we detected the MDA (a marker for oxidative stress and liver cell injury) and Hyp (a main component in collagen tissue) content in the liver tissue. We found that the level of MDA and Hyp in the hFSSC secretome group was significantly lower than the other two control groups (Fig. $1 \mathrm{~d}$ and e $p<0.01$ ). These findings suggest that hFSSC secretome effectively reduced $\mathrm{CCl}_{4}$-induced liver fibrosis in rats.

\section{hFSSC secretome reduced liver fibrosis through suppressing the EMT}

To further verify the roles of hFSSC secretome in the pathogenesis of liver fibrosis, we performed immunofluorescence staining of TGF- $\beta$ in HSCs, as TGF- $\beta 1$ is 


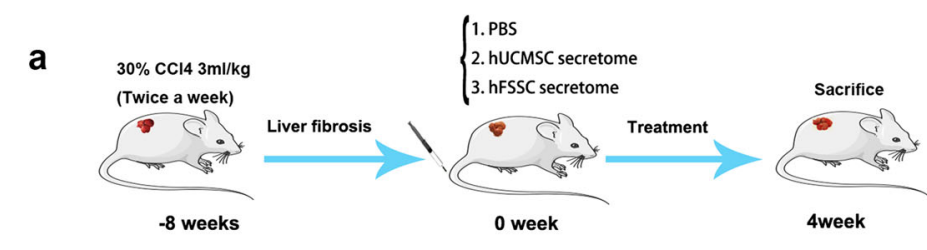

b
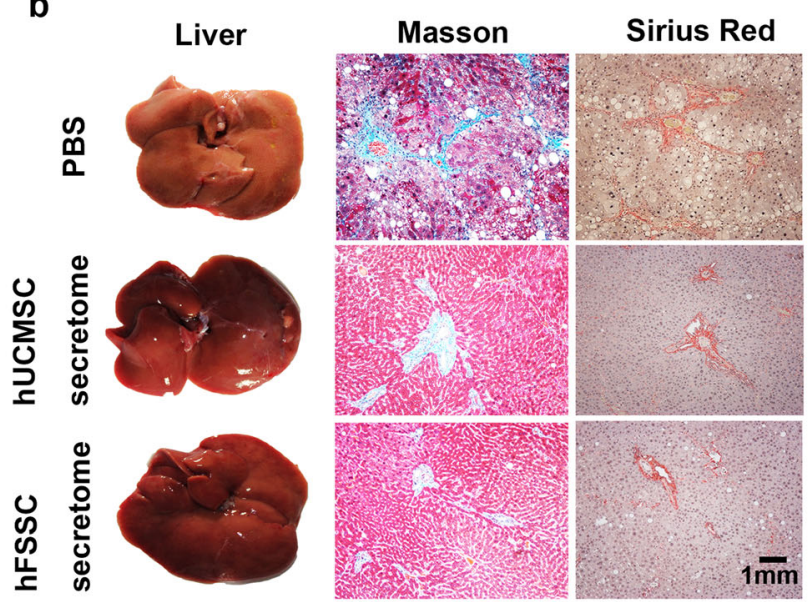

C

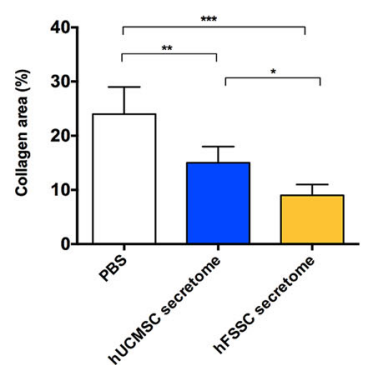

d

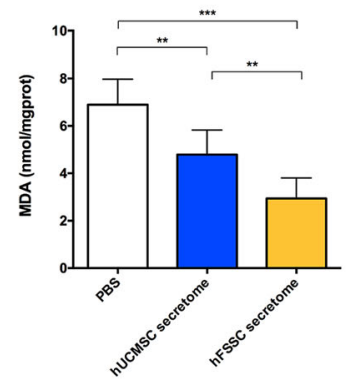

e

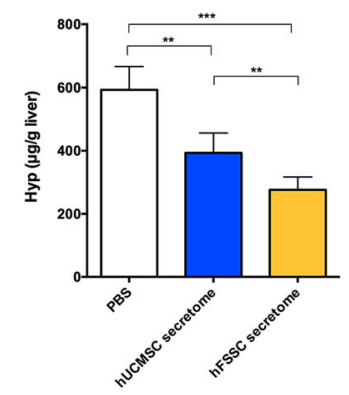

Fig. 1 hFSSC secretome reduced liver fibrosis in rats. a Experimental design. $\mathbf{b}$ The representative images of gross morphology, Masson, and Sirius red staining analysis of liver. Bar $=1 \mathrm{~mm}, n=10$ rats. c Quantitative analysis of the collagen area percentage at 4 weeks. d Quantitative analysis of hepatic MDA and Hyp content. ${ }^{*} p<0.05,{ }^{* *} p<0.01,{ }^{* * *} p<0.001 . n=10$; mean \pm SD

considered as a crucial mediator in tissue fibrosis and HSCs are one of the major effector cells in liver fibrosis. We found that the hFSSC secretome group reduced fluorescence intensity observably, compared to the other two control groups (Fig. 2a). In further study, we explore the effect of hFSSC secretome on EMT, and RT-qPCR analysis was used to examine the expression of EMTrelated indicators (E-cadherin, Snaill, Vimentin, FSP1, and $\alpha$-SMA in HSCs). Interestingly, our results found that hFSSC secretome treatment increased the epithelial marker of E-cadherin expression, while decreasing the transcription factors of Snail and mesenchymal marker (Vimentin, FSP1 and $\alpha$-SMA) expression, compared to the PBS group (Fig. 2b, $p<0.05$ ). Meanwhile, we also examined hFSSC secretome increased E-cadherin and decreased FSP1 and $\alpha$-SMA expression compared to the hUCMSC secretome group (Fig. $2 \mathrm{~b}, p<0.05$ ). These results suggest that hFSSC secretome reduced liver fibrosis through suppressing the EMT.

\section{hFSSC secretome improved liver functionality and promoted liver regeneration}

To explore the effect of hFSSC secretome on liver functionality, we performed the biochemical analyses. In comparison to the PBS group, hFSSC secretome group significantly reduced serum levels of ALT, AST, TBIL, $\gamma$ GT, and ALP (Fig. 3a-e, $p<0.05$ ). However, the serum level of TP in hFSSC secretome group was higher than that in PBS group (Fig. 3f, $p<0.05$ ). In addition, the hFSSC secretome group significantly reduced the serum 

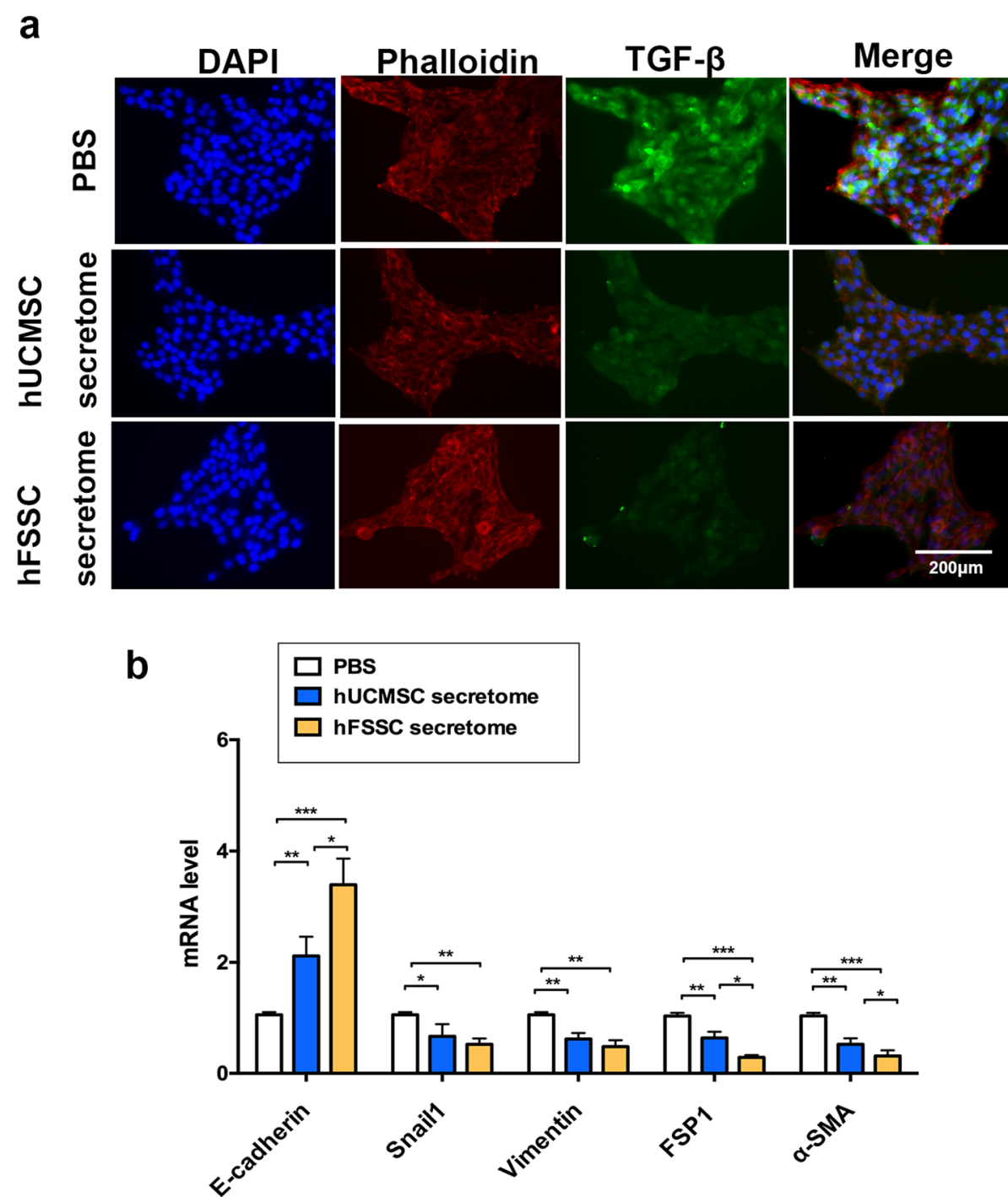

Fig. 2 hFSSC secretome reduced liver fibrosis through suppressing the EMT process. a Representative images of immunofluorescence staining performed for TGF- $\beta$ in HSCs. Bar $=200 \mu \mathrm{m}$. b Relative mRNA expression levels of EMT-related gene, included of E-cadherin, Snail1, Vimentin, FSP1, and a-SMA in HSCs. EMT, epithelial-mesenchymal transition; FSP1, fibroblast-specific protein 1; $a-S M A$, alpha smooth muscle actin. ${ }^{*} p<0.05$, ${ }^{* *} p<0.001,{ }^{* * *} p<0.0001 . n=10$, mean \pm SD

levels of TBIL and $\gamma$-GT compared to the hUCMSC secretome group (Fig. $3 c$ and $d, p<0.05$ ). These results suggest that hFSSC secretome effectively improved liver functionality.

Next, we performed IHC to assess the effects of the hFSSC secretome on the liver. $\alpha$-SMA is an important indicator of the occurrence and development of hepatic fibrosis. IHC results showed that the percentage of $\alpha$-SMApositive area in hFSSC secretome group (0.82\%) was significantly decreased compared to the PBS group (5.51\%, Fig. $4 \mathrm{a}$ and $\mathrm{b}, p<0.001)$. PCNA is a crucial indicator of cell proliferation. IHC results showed that the percentage of PCNA positive area in hFSSC secretome group (4.13\%) was significantly increased compared to the PBS group
(7.48\%, Fig. 4a and c, $p<0.01)$. Consist with the above results, the percentage of HNF- $4 \alpha$-positive area was significantly increased in the hFSSC secretome group (12.1\%), compared to the PBS group $(4.5 \%, p<0.01)$ as well as the hUC-MSCs group (8.2\%, Fig. $4 \mathrm{a}$ and $\mathrm{d}, p<0.05)$. The histological results indicated that hFSSC secretome effectively delayed the progression of liver fibrosis and promoted the liver regeneration.

\section{hFSSC secretome regulate the TGF- $\beta /$ Smad signal pathway}

To investigate the underlying mechanism of the effect of hFSSC secretome on liver fibrosis, we performed the Western blot and RT-qPCR to analyze the expression of 


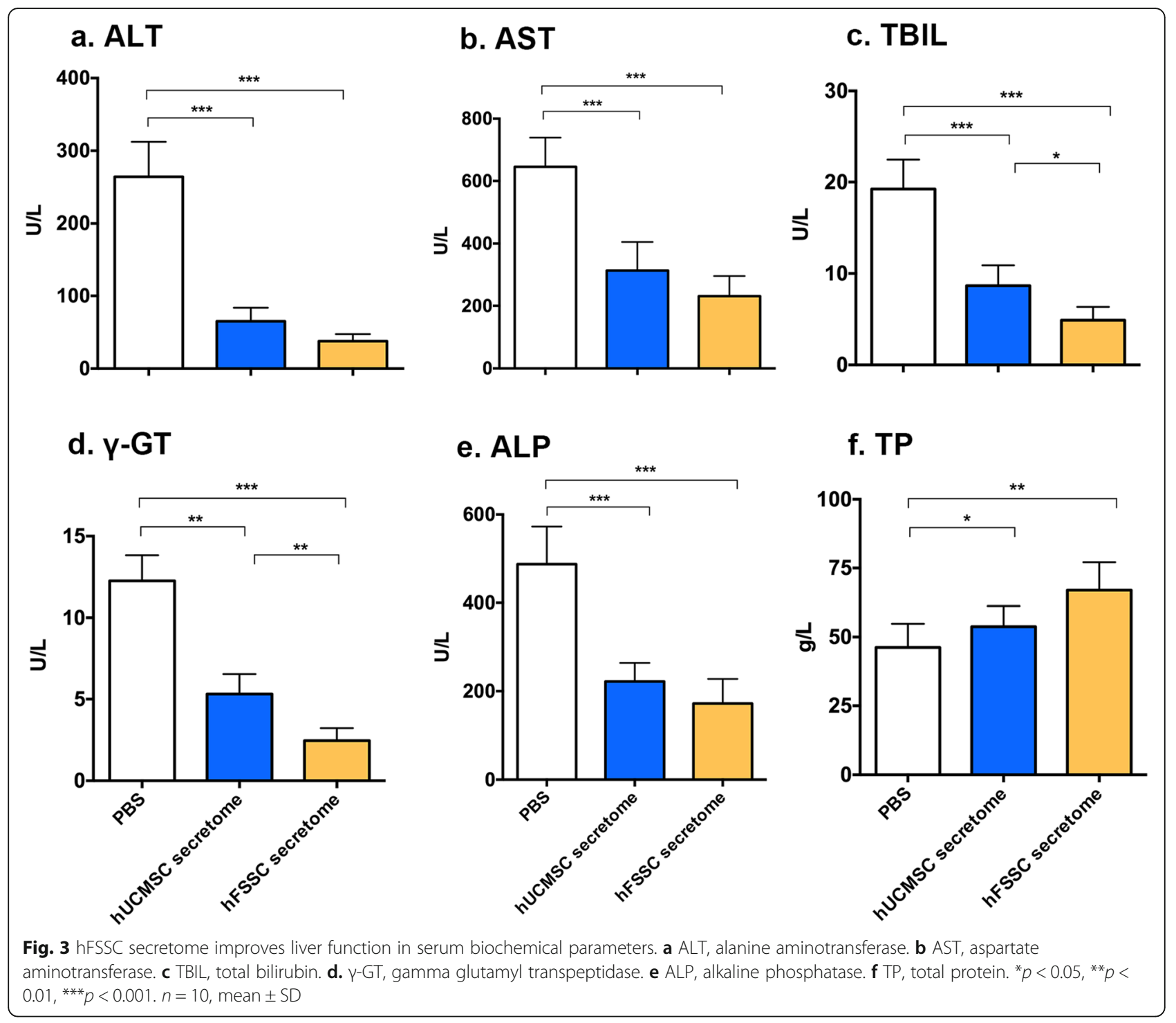

TGF- $\beta 1$, Smad2, Smad3, Smad7, and Collagen I in HSCs, as it is one of the major effector cells in liver fibrosis. We found that TGF- $\beta 1$, Smad2, Smad3, and Collagen I expression was significantly decreased in hFSSC secretome group, compared to that of the PBS group (Fig. 5a and b, $p<0.001$ ). However, we detected the Smad7 was significantly increased in the hFSSC secretome group, compared to that of the other two control groups (Fig. $5 \mathrm{a}$ and $\mathrm{b}, p<0.01$ ). Smad7 serves as a negative feedback regulator of TGF- $\beta 1 /$ Smad pathway, thereby protecting against TGF- $\beta 1$-mediated fibrosis (Fig. 6). These results suggest that hFSSC secretome effectively reduced liver fibrosis via regulating the TGF- $\beta$ / Smad signal pathway (Fig. 5).

\section{Discussion}

In this study, our results demonstrated that hFSSC secretome reduce liver fibrosis in rats. Moreover, our research illustrated that hFSSC secretome reduce liver fibrosis through suppressing the EMT and regulating the TGF- $\beta$ /Smad signal pathway in HSCs.

Recent evidence indicates that the mesenchymal stem cell secretome as an acellular regenerative therapy for liver disease [5, 14]. The use of MSC secretome has been shown to have anti-fibrotic effects [14]. Moreover, IV injection of hucMSC-EVs decreased liver fibrosis, reduced apoptosis, and mitigated liver damage induced by $\mathrm{CCl}_{4}$ in mice $[15,16]$. In our study, we found that hFSSC secretome effectively reduced $\mathrm{CCl}_{4}$-induced liver fibrosis and improved liver functionality in rats (Fig. 1). Moreover, hFSSC secretome treatment is more effective than that of hUCMSC secretome in resolving fibrosis, such as some indicators including collagen area, MDA, Hyp, TBIL, and $\gamma$-GT. The reasons that differences were found between hUCMSCs and hFSSC secretome may be reported that hFSSC secretome contains more biological 


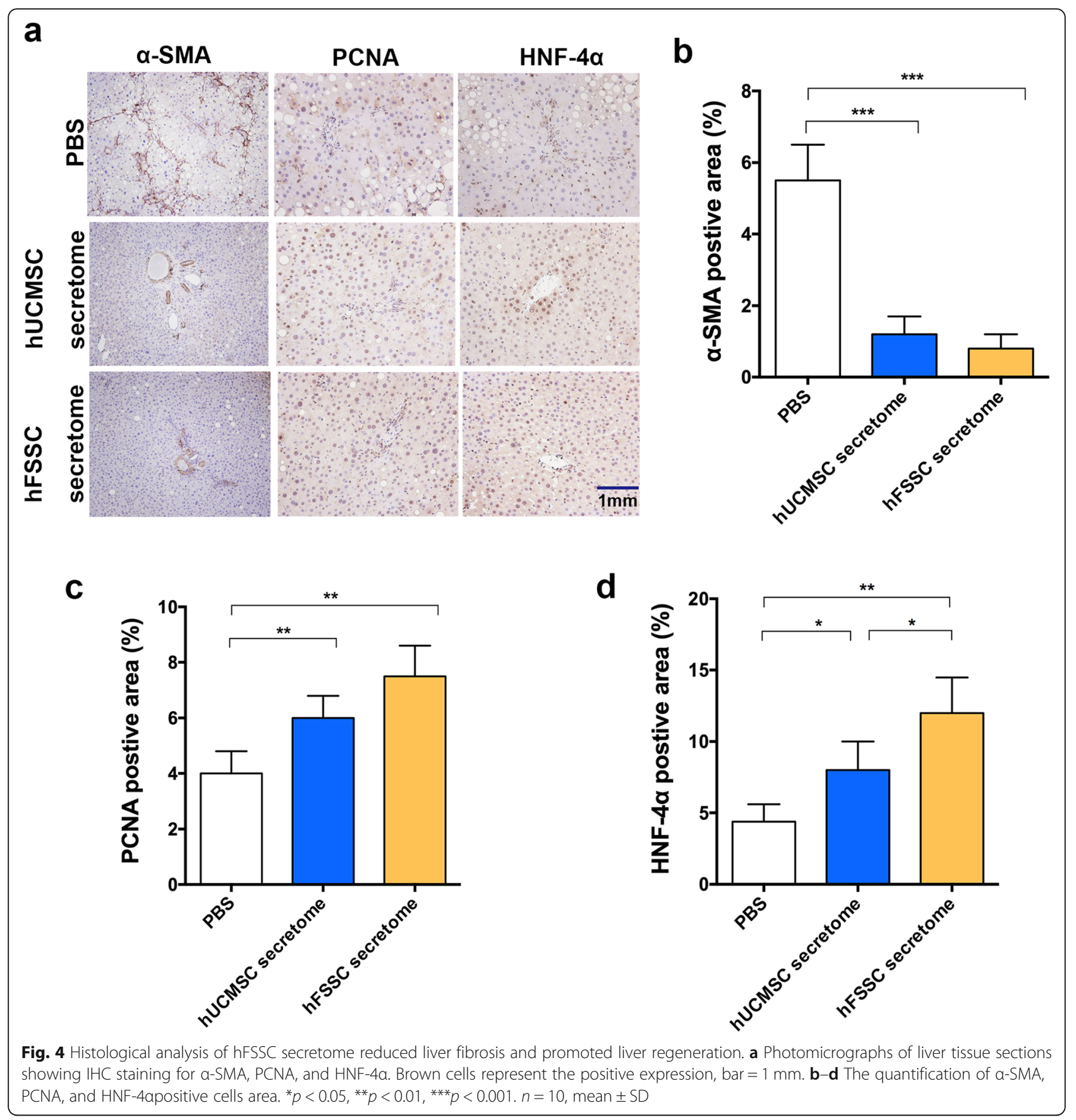

activity factors than hUCMSC secretome [13]. Our studies indicated that hFSSC secretom is an attractive emerging option for therapeutic applications as a therapeutic strategy for liver fibrosis.

Previous study have showed that mesenchymal stem cell secretome inhibit HSC activation and promoted liver regeneration [17-19]. HSCs stimulate the production of a large amount of collagen fibers to form liver fibrosis. Moreover, positive expression of $\alpha$-SMA can serve as a marker for HSC activation [20]. Our results demonstrate that hFSSC secretome decreased $\alpha$-SMA expression. PCNA is a crucial indicator of cell proliferation, and HNF- $4 \alpha$ is the master regulator of hepatic cell differentiation [15]. Our study demonstrates that the percentage of PCNA and HNF-4 $\alpha$-positive area was significantly increased after hFSSC secretome treatment. These results confirmed that hFSSC secretome promotes liver regeneration while reducing fibrosis. Despite our results confirming that hFSSC secretome had significantly reduced liver fibrosis and caused no detectable immunological 

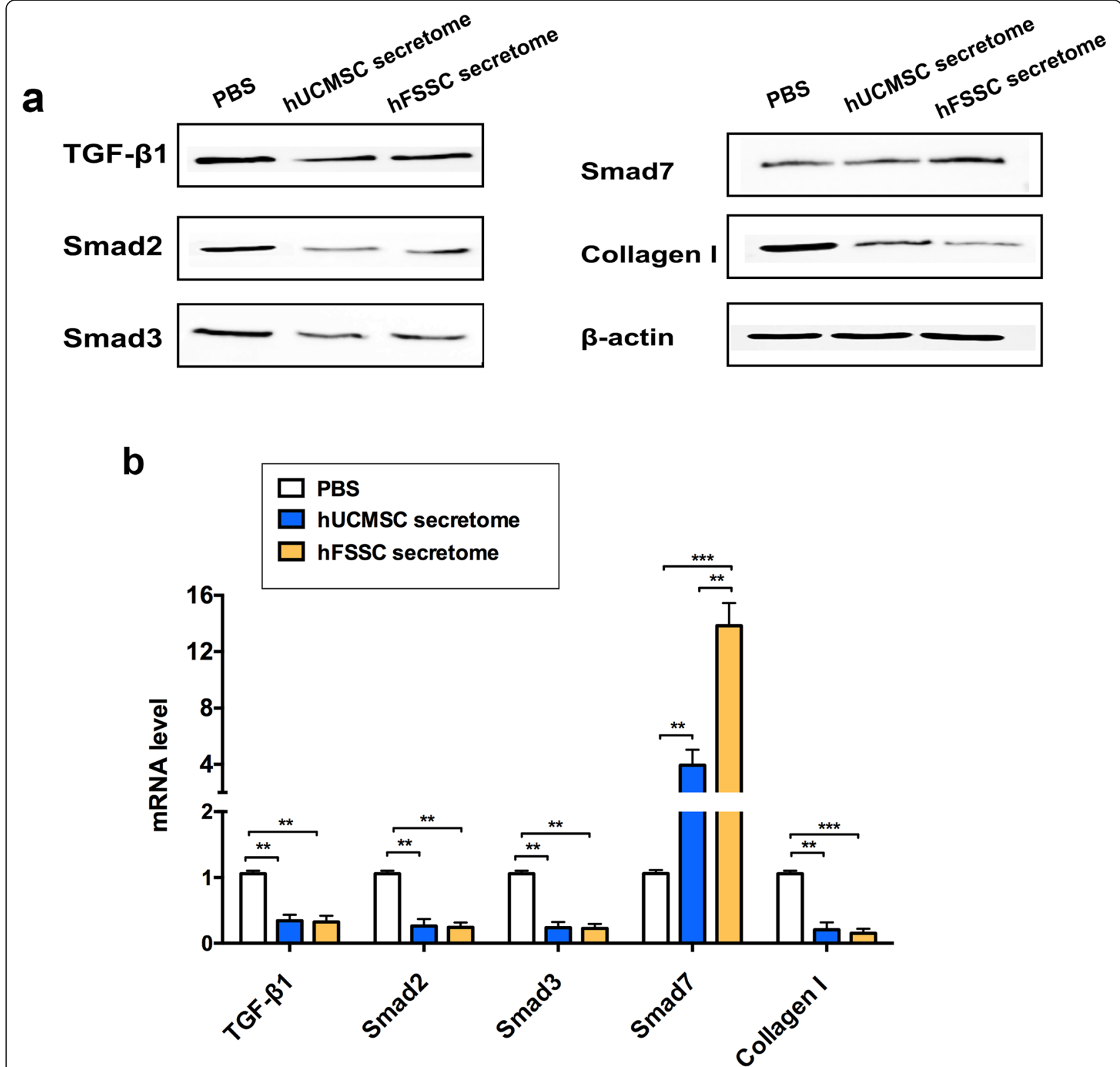

Fig. 5 hFSSC secretome inhibited the TGF- $\beta /$ Smad signaling pathway in HSCS. a Representative western blotting analysis the expression of TGF$\beta 1$, Smad2, Smad3, Smad7, and Collagen I in HSCs. b Relative mRNA expression levels of TGF- $\beta 1$, Smad2, Smad3, Smad7, and Collagen I in HSCs. ${ }^{*} p<0.001,{ }^{* *} p<0.001 . n=3$, mean \pm SD

responses, their exact mechanisms of action need further exploration. Moreover, study has reported that type 2 EMT is associated with tissue regeneration and organ fibrosis. After $\mathrm{CCl}_{4}$ injury in liver, inflammatory cytokines or chemical substances stimulate EMT to produce new collagen fibroblast pools to repair injury [5]. In present study, we demonstrated that hUCMSC secretome reduced liver fibrosis through suppressing the EMT process.

The MSC secretome secreted some growth factors and cytokines, such as hepatocyte growth factor (HGF), transforming growth factor beta isoform 3 (TGF- 33 ), and tumor necrosis factor-alpha (TNF- $\alpha$ ), and IL-10 can modulate cell signaling and processes involved in fibrogenesis and can attenuate liver fibrosis [5, 21]. Recently, fetal mesenchymal stem cell functional secretome analysis illustrates that $737 \pm 80$ protein identifications was obtained from the amniotic fluid-mesenchymal stem cell (AF-MSC) secretome; interestingly, it reveals that Annexin-A1 is important paracrine factor in hepatic regeneration [22]. However, previous study demonstrated that hFSSC presented with the characteristics of both MSC and embryonic stem cell (ESC) [13]. These may be the partial reason 


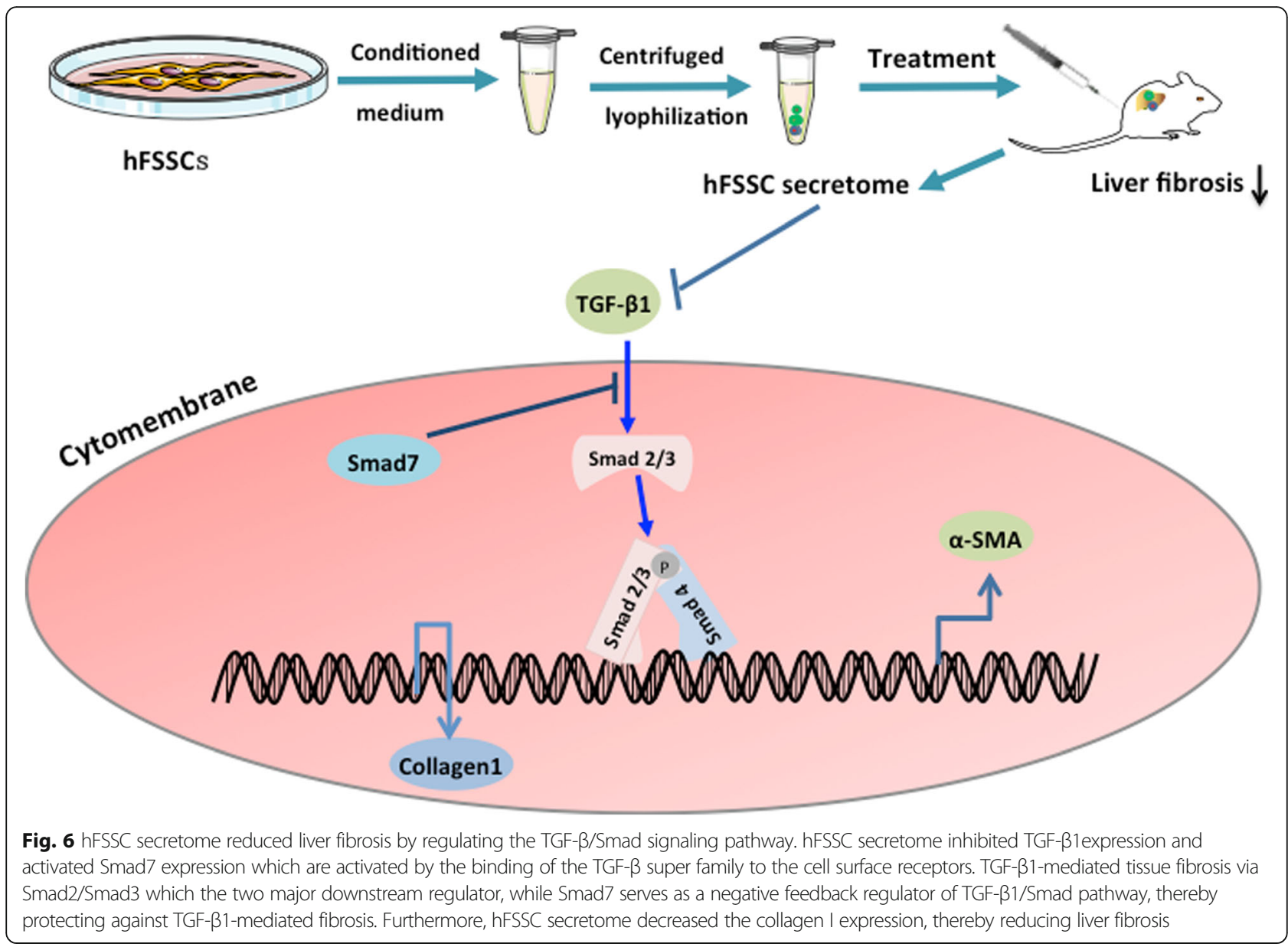

that hFSSC secretome can reduce liver fibrosis and has better effects than hUCMSC secretome in some indictors.

TGF- $\beta 1 /$ Smad pathway is an important pathogenic mechanism in tissue fibrosis [23, 24]. Studies have shown that TGF- $\beta 1$ is considered as a crucial mediator in tissue fibrosis and causes tissue scarring largely by activating its downstream small mother against decapentaplegic (Smad) signaling [25]. However, different TGF- $\beta$ signalings play different roles in fibrogenesis [24]. TGF- $\beta 1$ directly activates Smad signaling which triggers pro-fibrotic gene overexpression [26]. Increasing studies have demonstrated that dysregulation of TGF- $\beta 1 /$ Smad pathway was an important pathogenic mechanism in tissue fibrosis [27]. Smad2 and Smad3 are the two major downstream regulator that promote TGF- $\beta 1$-mediated tissue fibrosis, while Smad7 serves as a negative feedback regulator of TGF- $\beta 1 /$ Smad pathway, thereby protecting against TGF- $\beta 1$ mediated fibrosis [24]. Our findings proved that hFSSC secretome effectively reduced TGF- $\beta 1$, Smad2, Smad3, and Collagen I expression, moreover increasing Smad7 expression. It indicated that hFSSC secretome effectively reduced liver fibrosis via regulating the TGF- $\beta /$ Smad signal pathway (Fig. 6).

In conclusion, we successfully investigated the role of hFSSC secretome on cutaneous liver fibrosis. Our results demonstrated that hFSSC secretome can exert promoting effect of liver fibrosis via regulating the TGF- $\beta / \mathrm{Smad}$ signal pathway.

\section{Supplementary information}

Supplementary information accompanies this paper at https://doi.org/10 1186/s13287-020-01891-5.

Additional file 1. Table S1. Primers used for qRT-PCR

\section{Abbreviations}

hFSSC: Human fetal skin-derived stem cell; MSCs: Mesenchymal stem cells; hUCMSCs: Human umbilical cord mesenchymal stem cell; HSCs: Hepatic stellate cells; ALT: Alanine aminotransferase; AST: Aspartate aminotransferase; TBIL: Total bilirubin; $\gamma$-GT: Gamma glutamyl transpeptidase; ALP: Alkaline phosphatase; TP: Total protein; Hyp: Hydroxyproline; MDA: Malondialdehyde; RT-qPCR: Quantitative real-time PCR; a-SMA: Alpha-smooth muscle actin; HGF: Hepatocyte growth factor; EMT: Epithelial to mesenchymal transition; TNF-a: Tumor necrosis factor-alpha; ESC: Embryonic stem cell;

FSP1: Fibroblast-specific protein 1 


\section{Acknowledgements}

The authors thank Professor Chen, Changchun University of Traditional Chinese Medicine, for his assistance in animal experiment.

\section{Authors' contributions}

X.Y. carried out the cell culture and animal studies and helped to draft the manuscript. J.W. carried out animal studies and RT-qPCR. J.Z. carried out WB and performed the statistical analysis. X.R. conceived of the study and participated in its design and coordination and helped to draft the manuscript. The authors read and approved the final manuscript.

\section{Funding}

This work was supported by the National Natural Science Foundation of China (No.8762069).

\section{Availability of data and materials}

The datasets used and/or analyzed during the present study are available from the corresponding author on reasonable request.

\section{Ethics approval and consent to participate}

All the protocols and procedures were approved by the Animal Experiment Ethics Committee of the Changchun University of Traditional Chinese Medicine, China (Approval NO. XW201903167). The informed written consent form was approved by the Clinical Research Ethics Committee and signed by the donor before sample collection.

\section{Consent for publication}

Not applicable.

\section{Competing interests}

The author(s) declared no potential conflicts of interest with respect to the research, authorship, and/or publication of this article.

\section{Author details}

${ }^{1}$ Department of Anesthesiology, The Affiliated Hospital of Changchun University of Chinese Medicine, 1478 Gongnong Road, Changchun 130021 Jilin, China. ${ }^{2}$ Department of Gynecology, The Affiliated Hospital of Changchun University of Chinese Medicine, 1478 Gongnong Road, Changchun 130021, Jilin, China. ${ }^{3}$ Department of Radiology, The Third Hospital of Jilin University, 126 Xiantai St., Changchun 130033, Jilin, China. ${ }^{4}$ Department of Clinical Laboratory, The Affiliated Hospital of Changchun University of Chinese Medicine, 1478 Gongnong Road, Changchun 130021, Jilin, China. ${ }^{5}$ Neuroregeneration and Stem Cell Programs, Institute for Cell Engineering, The Johns Hopkins University School of Medicine, Baltimore, MD 21205, USA

Received: 30 June 2020 Revised: 5 August 2020

Accepted: 18 August 2020 Published online: 03 September 2020

\section{References}

1. Sun M, Kisseleva T. Reversibility of liver fibrosis. Clin Res Hepatol Gastroenterol. 2015;39(Suppl 1):S60-3.

2. Arriazu E, Ruiz de Galarreta M, Cubero FJ, Varela-Rey M, Perez de Obanos MP, Leung TM, Lopategi A, Benedicto A, Abraham-Enachescu I, Nieto N. Extracellular matrix and liver disease. Antioxid Redox Signal. 2014;21(7): 1078-97.

3. Elpek GO. Cellular and molecular mechanisms in the pathogenesis of liver fibrosis: an update. World J Gastroenterol. 2014;20(23):7260-76.

4. Konala VB, Mamidi MK, Bhonde R, Das AK, Pochampally R, Pal R. The current landscape of the mesenchymal stromal cell secretome: a new paradigm for cell-free regeneration. Cytotherapy. 2016;18(1):13-24

5. Driscoll J, Patel T. The mesenchymal stem cell secretome as an acellular regenerative therapy for liver disease. J Gastroenterol. 2019;54(9):763-73.

6. Rong X, Liu J, Yao X, Jiang T, Wang Y, Xie F. Human bone marrow mesenchymal stem cells-derived exosomes alleviate liver fibrosis through the Wnt/beta-catenin pathway. Stem Cell Res Ther. 2019;10(1):98.

7. Moerkamp AT, Lodder K, van Herwaarden T, Dronkers E, Dingenouts CK, Tengstrom FC, van Brakel TJ, Goumans MJ, Smits AM. Human fetal and adult epicardial-derived cells: a novel model to study their activation. Stem Cell Res Ther. 2016;7(1):174.
8. Ishii T, Eto K. Fetal stem cell transplantation: past, present, and future. World I Stem Cells. 2014:6(4):404-20.

9. Zhang ZY, Teoh SH, Hui JH, Fisk NM, Choolani M, Chan JK. The potential of human fetal mesenchymal stem cells for off-the-shelf bone tissue engineering application. Biomaterials. 2012;33(9):2656-72.

10. Xu J, Wang B, Sun Y, Wu T, Liu Y, Zhang J, Lee WY, Pan X, Chai Y, Li G. Human fetal mesenchymal stem cell secretome enhances bone consolidation in distraction osteogenesis. Stem Cell Res Ther. 2016;7(1):134.

11. Chan J, O'Donoghue K, de la Fuente J, Roberts IA, Kumar S, Morgan JE, Fisk NM. Human fetal mesenchymal stem cells as vehicles for gene delivery. Stem cells (Dayton). 2005;23(1):93-102.

12. Wang $X$, Jiao $Y$, Pan $Y$, Zhang L, Gong H, Qi Y, Wang M, Gong H, Shao M, Wang $X$, et al. Fetal dermal mesenchymal stem cell-derived exosomes accelerate cutaneous wound healing by activating Notch signaling. Stem Cells Int. 2019:2019:2402916.

13. Rong $X$, Li J, Yang Y, Shi L, Jiang T. Human fetal skin-derived stem cell secretome enhances radiation-induced skin injury therapeutic effects by promoting angiogenesis. Stem Cell Res Ther. 2019;10(1):383.

14. Jang YJ, An SY, Kim JH. Identification of MFGE8 in mesenchymal stem cell secretome as an anti-fibrotic factor in liver fibrosis. BMB Rep. 2017:50(2):589.

15. Li T, Yan Y, Wang B, Qian H, Zhang X, Shen L, Wang M, Zhou Y, Zhu W, Li $W$, et al. Exosomes derived from human umbilical cord mesenchymal stem cells alleviate liver fibrosis. Stem Cells Dev. 2013;22(6):845-54.

16. Lou G, Chen Z, Zheng M, Liu Y. Mesenchymal stem cell-derived exosomes as a new therapeutic strategy for liver diseases. Expreriment Mol Med. 2017; 49(6):e346.

17. Li Q, Zhou X, Shi Y, Li J, Zheng L, Cui L, Zhang J, Wang L, Han Z, Han Y, et al. In vivo tracking and comparison of the therapeutic effects of MSCs and HSCs for liver injury. PLoS One. 2013;8(4):e62363.

18. Berardis S, Dwisthi Sattwika P, Najimi M, Sokal EM. Use of mesenchymal stem cells to treat liver fibrosis: current situation and future prospects. World J Gastroenterol. 2015:21(3):742-58.

19. Eom YW, Shim KY, Baik SK. Mesenchymal stem cell therapy for liver fibrosis. Korean J Int Med. 2015:30(5):580-9.

20. Bataller R, Brenner DA. Liver fibrosis. J Clin Investig. 2005;115(2):209-18.

21. Alfaifi M, Eom YW, Newsome PN, Baik SK. Mesenchymal stromal cell therapy for liver diseases. J Hepatol. 2018;68(6):1272-85.

22. Zagoura D, Trohatou O, Makridakis M, Kollia A, Kokla N, Mokou M, Psaraki A, Eliopoulos AG, Vlahou A, Roubelakis MG. Functional secretome analysis reveals Annexin-A1 as important paracrine factor derived from fetal mesenchymal stem cells in hepatic regeneration. EBioMedicine. 2019;45: 542-52.

23. Hu HH, Chen DQ, Wang YN, Feng YL, Cao G, Vaziri ND, Zhao YY. New insights into TGF-beta/Smad signaling in tissue fibrosis. Chem Biol Interact. 2018;292:76-83.

24. Walton $\mathrm{KL}$, Johnson KE, Harrison CA. Targeting TGF-beta mediated SMAD signaling for the prevention of fibrosis. Front Pharmacol. 2017:8:461.

25. Mokoena D, Dhilip Kumar SS, Houreld NN, Abrahamse H. Role of photobiomodulation on the activation of the Smad pathway via TGF-beta in wound healing. J Photochem Photobiol B. 2018;189:138-44.

26. Grotendorst GR. Connective tissue growth factor: a mediator of TGF-beta action on fibroblasts. Cytokine Growth Factor Rev. 1997;8(3):171-9.

27. Lindert S, Wickert L, Sawitza I, Wiercinska E, Gressner AM, Dooley S, Breitkopf K. Transdifferentiation-dependent expression of alpha-SMA in hepatic stellate cells does not involve TGF-beta pathways leading to coinduction of collagen type I and thrombospondin-2. Matrix Biol. 2005;24(3):198-207.

\section{Publisher's Note}

Springer Nature remains neutral with regard to jurisdictional claims in published maps and institutional affiliations. 